\section{Childhood pneumonia: the role of viruses}

\author{
H J Zar, ${ }^{1,2}$ F P Polack ${ }^{3,4}$
}

The development and availability of improved vaccines, particularly the pneumococcal (PCV) and Haemophilus influenzae type $\mathrm{b}$ (Hib) conjugate vaccines have substantially reduced the incidence of childhood pneumonia and severe pneumonia associated with these pathogens over the last decade. ${ }^{1}$ However, despite a declining pneumonia incidence and high rates of immunisation, pneumonia remains the leading cause of childhood mortality globally and a major cause of hospitalisation. ${ }^{2}$

Defining the aetiology of pneumonia has become crucial to develop appropriate management strategies, and guide development of new vaccines. Further, the development of better methods for specimen collection and of molecular diagnostics has provided more sensitive techniques to define potential aetiological agents. ${ }^{3}$ However, assigning aetiology may be challenging as it can be difficult to distinguish colonising from pathogenic organisms in respiratory specimens, blood culture rarely is positive and pneumonia, especially severe disease, may frequently be due to multiple copathogens.

In this issue of Thorax, Rhedin and colleagues report the association of viral pathogens and radiological pneumonia in Swedish children (median age 21 months) using a matched case-control study conducted over three winter seasons. ${ }^{4}$ PCV immunisation was well established before the study began. Healthy control children were randomly selected during routine visits; aetiological diagnosis was investigated using a quantitative PCR assay for 15 viruses performed on nasopharyngeal aspirates. There are several notable findings. First, viruses were detected in $>80 \%$ of cases and in approximately $60 \%$ of controls. Second, in multivariate analysis, respiratory syncytial virus (RSV) (adjusted OR (aOR) 21.3), influenza virus (aOR

${ }^{1}$ Department of Paediatrics and Child Health, Red Cross War Memorial Children's Hospital, Cape Town, South Africa; ${ }^{2} \mathrm{MRC}$ Unit on Child and Adolescent Health, University of Cape Town, Cape Town, South Africa; ${ }^{3}$ Department of Pediatrics, Vanderbilt University, Nashville, Tennessee, USA; ${ }^{4}$ Fundacion INFANT, Buenos Aires, Argentina

Correspondence to Professor H J Zar, Department of Paediatrics and Child Health, Red Cross War Memorial Children's Hospital, 5th Floor, ICH Building, Cape Town 7700, South Africa; heather.zar@uct.ac.za
13.8) or human metapneumovirus (hMPV) (aOR 14.9) were most strongly associated with pneumonia, with RSV being most commonly detected. However, for other viruses, including rhinovirus or adenovirus, there were no significant differences in detection rates between cases and controls, indicating that aetiology cannot be attributed to these. Third, coinfection with different viruses was common, occurring in approximately one-third of cases and 21\% of controls. Finally, viral load was significantly higher for RSV and for hMPV in cases compared with controls. A limitation of the study is the lack of concomitant bacterial data, as bacterial-viral interactions and coinfection may be common in childhood pneumonia and associated with more severe disease. This also reflects the difficulty of establishing bacterial aetiology in childhood pneumonia as current tests on respiratory specimens, primarily induced sputum or nasopharyngeal samples, lack sensitivity to distinguish aetiological from colonising bacteria.

These results are consistent with a recent large case-control study of children (median age 2 years) hospitalised for pneumonia in the USA over 2.5 years. ${ }^{5}$ This study found a virus (detected by PCR on nasopharyngeal or oropharyngeal swabs) in over $70 \%$ of cases, a bacterial agent (defined as a positive culture for selected bacteria on blood, endotracheal aspirate, bronchoalveolar lavage or pleural fluid or a positive PCR on pleural fluid or blood) in $15 \%$ and multiple pathogens in just over a quarter. RSV, found in $28 \%$ of cases, was the most common pathogen detected, with the greatest burden in children under 2 years of age. Influenza virus and hMPV were also more commonly found in cases than controls. However, in contrast to the Swedish study, adenovirus, parainfluenza virus and coronavirus were more common in cases, although they represented a small proportion of the aetiological agents detected in the study. Perhaps, some of these differences are explained by a larger and consequently better powered North American study. Alternatively, variations may be associated with the selection of controls. While controls in Sweden were closely matched by season and age, a convenience sample of asymptomatic children undergoing same-day elective surgery was selected as controls in the US study. Limitations to interpreting the US casecontrol data include much fewer controls $(n=521)$ than cases $(n=2012)$, controls only enrolled at selected sites, the significantly older age of controls compared with cases and the lack of data on the exact prevalence of viruses (other than rhinovirus) in controls.

These studies indicate that RSV is a major cause of pneumonia in the era of conjugate vaccines for bacterial pathogens. However, they also highlight the limitations of current diagnostic strategies, particularly the poor sensitivity of current tests for bacterial aetiology and the potential for incorrectly assigning aetiology based on molecular diagnostics. They also provide further data on the complexity of ascribing pneumonia aetiology, showing interactions between multiple potential pathogens. Despite these limitations, the emerging data indicate that a key strategy for reducing the burden of childhood pneumonia lies in prevention of RSV disease in young children.

A limitation of these studies may be their lack of generalisability to children in low and middle-income countries (LMICs) where the predominant burden of childhood pneumonia exists, and where there is a much higher burden of bacterial disease and lower rates of conjugate vaccine uptake. ${ }^{1}$ Further, in these settings, the highest incidence of pneumonia and of severe disease occurs in children under 1 year of age, in which different pathogens may predominate. $^{2}$ More high quality aetiological data are needed from children with pneumonia in LMIC settings. Nevertheless, epidemiological and cross-sectional studies of children with pneumonia in LMICs, including Africa, South East Asia and Latin America, performed prior to the availability of PCV, also indicate a high incidence of RSV, with much higher case-fatality rates in these settings compared with high-income countries. $^{6-9}$ In 2005, RSV was estimated to cause approximately 34 million episodes of acute lower respiratory tract infections (ALRI) in children under 5 years of age or $22 \%$ of all ALRI; $10 \%$ of episodes resulted in severe illness and hospitalisation, and $99 \%$ of deaths occurred in LMICs. $^{9}$ Further, a recent birth cohort study in a poor peri-urban area of South Africa, the Drakenstein Child Health Study, reported a high incidence of pneumonia and severe pneumonia in the first few months of life despite a strong immunisation programme, including PCV13. ${ }^{10}$ Increasing uptake of PCV in LMICs may further increase the relative contribution of RSV to the burden 
of pneumonia. However, in these settings, testing for respiratory viruses in infants and young children with severe or fatal pneumonia is infrequent for several reasons such as the absence of therapeutic alternatives and the cost and lack of molecular diagnostic tests in facilities serving low-income populations. Therefore, studies often underestimate the burden of RSV infection in this context, unless specifically designed to consider it. The development of a new RSV vaccine with a strategy to immunise pregnant women so as to protect infants in early life is a promising, novel intervention with potential to reduce this burden. ${ }^{11}$

These data also raise questions about the optimal management of childhood pneumonia and use of antibiotics. The reduction of $\mathrm{Hib}$ and pneumococcal pneumonia through conjugate vaccines underscores the need to reconsider the empirical treatment of pneumonia in settings where there are strong national immunisation programmes. With high coverage of $\mathrm{Hib}$ conjugate vaccine, $\mathrm{Hib}$ is unlikely to contribute to a substantial proportion of pneumonia cases. ${ }^{1}$ However, the importance of pneumococcus as an aetiological agent may vary depending on the prevalence of non-vaccines serotypes causing disease. While case management with antibiotics for pneumonia or severe pneumonia in the WHO Integrated Management of Childhood Illness (IMCI) programme has been a highly effective strategy for reducing mortality prior to widespread conjugate vaccine availability, defining the residual burden and identifying clinical or laboratory features that distinguish bacterial from viral pathogens will be important before any change in pneumonia strategy can be implemented globally.

Contributors All authors contributed to the writing of this editorial.

Funding $\mathrm{HZ}$ receives funding for pneumonia research in children from the Bill and Melinda Gates Foundation (OPP1017641), the National Institutes of Health (U01Al110466), Medical Research Council of South Africa and the National Research Foundation South Africa. FPP receives funding for research in acute respiratory infections and infant mortality from the Bill and Melinda Gates Foundation, the National Institutes of Health (Al-104853) and the Thrasher Research Fund. FP receives funding for research in acute respiratory infections and infant mortality from the Bill and Melinda Gates Foundation (OPP1084347), the National Institutes of Health (Al-104853) and the Thrasher Research Fund (9133).

Competing interests None declared.

Provenance and peer review Commissioned; internally peer reviewed.

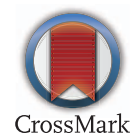

To cite Zar HJ, Polack FP. Thorax 2015;70:811-812.

Published Online First 19 June 2015

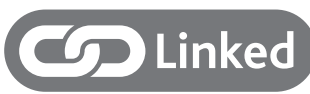

http://dx.doi.org/10.1136/thoraxjnl-2015-206933

Thorax 2015;70:811-812.

doi:10.1136/thoraxjnl-2015-207320

\section{REFERENCES}

1 Zar HJ, Madhi SA, Aston SJ, et al. Pneumonia in low and middle income countries-progress and challenges. Thorax 2013:68:1052-6.

2 Liu L, Oza S, Hogan D, et al. Global, regional, and national causes of child mortality in 2000-13, with projections to inform post-2015 priorities: an updated systematic analysis. Lancet 2015;385:430-40.

3 Murdoch DR, O'Brien KL, Driscoll AJ, et al. Pneumonia Methods Working Group; PERCH Core Team. Laboratory methods for determining pneumonia etiology in children. Clin Infect Dis 2012;54(Suppl 2):S146-52.

4 Rhedin S, Lindstrand A, Hjelmgren A, et al. Respiratory viruses associated with communityacquired pneumonia in children: matched casecontrol study. Thorax 2015;70:847-53.

5 Jain S, Williams DJ, Arnold SR, et al. Community-acquired pneumonia requiring hospitalization among US children. N Eng/ I Med 2015;372:835-45.

6 Berkley JA, Munywoki P, Ngama M, et al. Viral etiology of severe pneumonia among Kenyan infants and children. JAMA 2010;303:2051-7.

7 Nasreen S, Luby SP, Brooks WA, et al. Population-based incidence of severe acute respiratory virus infections among children aged $<5$ years in rural Bangladesh, June-October 2010. PLoS ONE 2014;9:e89978.

8 Naorat S, Chittaganpitch M, Thamthitiwat S, et al. Hospitalizations for acute lower respiratory tract infection due to respiratory syncytial virus in Thailand, 2008-2011. J Infect Dis 2013;208(Suppl 3): S238-45.

9 Nair H, Nokes DJ, Gessner BD, et al. Global burden of acute lower respiratory infections due to respiratory syncytial virus in young children: a systematic review and meta-analysis. Lancet 2010;375:1545-55.

10 Le Roux D, Myer L, Nicol MP, et al. The incidence and severity of childhood pneumonia in the first year of life in a South African birth cohort: the Drakenstein Child Health Study. Lancet Global Health 2015;3:e95-103.

11 Guvenel AK, Chiu C, Openshaw PJ. Current concepts and progress in RSV vaccine development. Expert Rev Vaccines 2014;13:333-44. 